\title{
INFLUÊNCIA DA RELAÇÃO SUPERFÍCIE/VOLUME E DO TEMPO DE FRITURA SOBRE AS ALTERAÇÕES DA MISTURA AZEITE DE DENDE-ÓLEO DE SOJA
}

\author{
Factors influence of surface/volume ratio and frying time on the alterations to the \\ "Dendê" and soybean oil mixture
}

\author{
Cassia Roberta Malacrida ${ }^{1}$, Neuza Jorge ${ }^{2}$
}

\section{RESUMO}

Objetivou-se com este estudo determinar a influência dos fatores relação superfície/volume (S/V) e tempo de fritura sobre a alteração da mistura azeite de dendê-óleo de soja em frituras descontínuas de batatas chips. Foram realizados dois procedimentos de fritura com relações $\mathrm{S} / \mathrm{V}$ de 0,5 e $1,0 \mathrm{~cm}^{-1}$ e temperatura média de $180^{\circ} \mathrm{C}$. O óleo foi aquecido por um período de 7,25 horas. As amostras obtidas durante o processo de fritura foram analisadas pelas determinações de índice de peróxidos, compostos polares totais, ácidos graxos livres e índice de refração. Todas as determinações analíticas foram influenciadas pelo tempo de fritura, verificando-se aumentos significativos destes parâmetros ao longo do processo de fritura. O fator relação S/V apresentou influência sobre os valores dos índices de peróxidos e de refração. A mistura azeite de dendê-óleo de soja apresentou alteração acima do limite recomendado para óleos de fritura (compostos polares totais $\geq 25 \%$ ) a partir de 6,25 horas de fritura.

Termos para indexação: Óleos vegetais, frituras descontínuas, batatas chips.

\begin{abstract}
The purpose of this study was to determine the factors influence of surface/volume ratio $(\mathrm{S} / \mathrm{V})$ and frying time on the alteration to the "dendê" and soybean oil mixture during discontinuous frying of potatoes chips. Two frying procedures with surface/ volume ratios $(\mathrm{S} / \mathrm{V})$ of 0.5 and $1.0 \mathrm{~cm}^{-1}$ at temperature of $180^{\circ} \mathrm{C}$ were done. The oil was heated up for 7.25 hours. Oil samples obtained during the frying process were assessed for peroxide value, total polar compounds, free fatty acids and refractive index. All analytical determinations were influenced by frying time, verifying significative increases in the values during frying process. The factor S/V ratio showed influence on the peroxide values and refractive index. "Dendê" and soybean oil mixture showed alteration above the recommended limits for frying oils (total polar compounds $\geq 25 \%$ ) after 6.25 hours of frying.
\end{abstract}

Index terms: Vegetable oils, discontinuous frying, chips.

(Recebido para publicação em 27 de abril de 2004 e aprovado em 4 de abril de 2006)

\section{INTRODUÇÃO}

Durante o processo de fritura o óleo interage com o ar, a água e outros componentes do alimento que está sendo frito, originando uma grande diversidade de reações químicas, tais como hidrólise, oxidação e polimerização da molécula de triacilglicerol (SANIBAL \& MANCINI-FILHO, 2002).

Os produtos de alteração, resultantes da falta de um controle adequado do processo de fritura, afetam a qualidade nutricional e sensorial do alimento frito, podendo ainda causar danos à saúde do consumidor uma vez que óleos e gorduras aquecidos a altas temperaturas podem se oxidar e originar substâncias potencialmente tóxicas (NAWAR, 1996; TYAGI \& VASISHTHA, 1996).

Segundo Nawar (1996), as mudanças físicoquímicas que ocorrem em óleos utilizados por longos períodos são influenciadas por um grande número de parâmetros: composição do óleo e do alimento, tempo e temperatura de fritura, presença de contaminantes metálicos, tipo de aquecimento, relação superfície/volume, reposição com óleo novo e presença de antioxidantes.

A crescente utilização de óleos comestíveis para a preparação de produtos fritos tem levado a um controle mais rigoroso dos óleos de fritura. Existem numerosos métodos analíticos que podem ser utilizados para avaliar a deterioração dos óleos de fritura, tais como compostos polares totais, índice de peróxidos, acidez livre, índice de iodo e testes colorimétricos (MASSON et al., 1997; ROSSELL, 1986). A quantificação de compostos polares totais corresponde à medida da alteração total dos óleos e é mais eficaz, uma vez que fornece informações mais específicas do nível de degradação dos mesmos. A utilização do método de Waltking \& Wessels (1981) para a determinação de compostos polares totais é recomendada como método de referência para o descarte de óleos em muitos países (FIRESTONE, 1996). 
No Brasil, as frituras descontínuas são bastante utilizadas tanto no preparo doméstico de alimentos quanto em restaurantes e lanchonetes. O óleo é utilizado um elevado número de vezes com mínima reposição, o que pode originar elevados níveis de alteração (DAMY \& JORGE, 2003). No entanto, o Brasil ainda não possui regulamentação para o monitoramento e descarte de óleos de fritura. Tais fatos demonstram a importância de estudos para conhecer o comportamento de óleos vegetais em processos de frituras descontínuas e conhecer o grau de alteração dos mesmos.

A determinação do ponto de descarte dos óleos de fritura é importante, uma vez que implica em maior custo quando o óleo é descartado muito cedo e na perda da qualidade do alimento frito quando descartado tardiamente. Alguns países, entre eles Bélgica, França, Alemanha, Suíça, Holanda, Estados Unidos e Chile, criaram leis e regulamentações que estabelecem para o descarte de óleos de fritura valores superiores a $25 \%$ para compostos polares totais; superiores a $1 \%$ para ácidos graxos livres e superiores a $15 \mathrm{meq} / \mathrm{kg}$ de amostra para o índice de peróxidos (FIRESTONE et al., 1991; MONFERRER \& VILLALTA, 1993).

Neste contexto, com o presente trabalho, buscouse determinar a influência dos fatores relação superfície/ volume e tempo de fritura sobre a alteração da mistura azeite de dendê-óleo de soja em frituras descontínuas de batatas chips.

\section{MATERIAL E MÉTODOS}

\section{Amostras}

Para os ensaios de fritura descontínua de batatas utilizou-se uma mistura de azeite de dendê-óleo de soja na proporção de 1:1, marca Cepêra, embalada em garrafa PET de $900 \mathrm{~mL}$, adquirida no comércio local. Os ensaios foram conduzidos em um recipiente de alumínio, capacidade de 2 L, contendo 0,5 e 1,0 litro e relação superfície/volume de 1,0 e $0,5 \mathrm{~cm}^{-1}$, respectivamente. A temperatura empregada foi em torno de $180^{\circ} \mathrm{C}$.

As relações $\mathrm{S} / \mathrm{V}$ de 0,5 e $1,0 \mathrm{~cm}^{-1}$ inicialmente utilizadas neste trabalho correspondem a frituras realizadas em fritadeiras e frigideiras, atingindo ao final dos ensaios de fritura, valores de 0,8 e $2,6 \mathrm{~cm}^{-1}$, respectivamente. A relação $\mathrm{S} / \mathrm{V}$ foi calculada dividindo-se a área superficial do óleo em contato com o ar atmosférico dentro do recipiente pelo volume de óleo utilizado em cada ensaio. O número de frituras foi selecionado, levando-se em consideração os valores da temperatura e relação $\mathrm{S} / \mathrm{V}$, com o objetivo de se obter alterações similares às que se encontram ocasionalmente nos óleos de fritura.

Quinze lotes com aproximadamente $100 \mathrm{~g}$ de batatas da variedade binje cortadas em fatias (cerca de $0,3 \mathrm{~cm}$ de espessura) foram fritos por um período de 5 minutos. Para cada lote foram empregados intervalos de 25 minutos entre cada operação de fritura, nos quais o aquecimento da mistura foi interrompido por 20 minutos, sendo os cinco minutos restantes utilizados para reaquecimento. A mistura azeite de dendê-óleo de soja foi aquecida, para cada ensaio, por um período total de 7,25 horas sem qualquer reposição com óleo fresco.

Antes de dar início aos ensaios de fritura, a mistura azeite de dendê:óleo de soja foi submetida a um aquecimento por um período de tempo de 10 minutos para o estabelecimento de controle de temperatura no recipiente de alumínio. Amostras da mistura $(25 \mathrm{~mL})$ foram tomadas após 0,$25 ; 1,25 ; 2,25 ; 3,25 ; 4,25 ; 5,25 ; 6,25$ e 7,25 horas de fritura. Foram retiradas também amostras do óleo original para análise. Todas as amostras foram armazenadas em recipientes de vidro âmbar à temperatura de, aproximadamente, $-18^{\circ} \mathrm{C}$ para evitar posteriores alterações oxidativas incontroláveis.

\section{Determinações analíticas}

- Índice de peróxidos, expressos em miliequivalentes de oxigênio ativo por kg de óleo, conforme método da AOCS Cd 8-53 (AOCS, 1993);

- Compostos polares totais, expressos em porcentagem, obtidos por cromatografia em coluna, conforme o método proposto por Waltking \& Wessels (1981) com uma pequena modificação: o uso de hexano-éter etílico 90:10, ao invés de 87:13, para produzir uma melhor separação da fração não polar (DOBARGANES et al., 1984);

- Ácidos graxos livres, expressos em ácido oléico (\%), conforme método da AOCS Cd 3d-63 (AOCS, 1993);

- Índice de refração, determinado a $40^{\circ} \mathrm{C}$ em Refratômetro de Abbé, conforme método da AOCS Cc 7-25 (AOCS, 1993).

\section{Análise estatística}

Os resultados dos experimentos, em duas repetições, foram submetidos a análises estatísticas, utilizando-se esquema fatorial $2 \times 8$, sendo duas relações superfície/volume $\left(0,5\right.$ e $\left.1,0 \mathrm{~cm}^{-1}\right)$ e oito tempos de fritura $(0,25 ; 1,25 ; 2,25 ; 3,25 ; 4,25 ; 5,25 ; 6,25$ e 7,25 horas $)$. Os resultados obtidos das determinações analíticas, em duplicatas, foram comparados pelo teste de Tukey a $1 \mathrm{e}$ $5 \%$ de probabilidade (GOMES, 2000). 


\section{RESULTADOS E DISCUSSÃO}

As características físico-químicas iniciais da mistura azeite de dendê-óleo de soja (1:1) são apresentadas na Tabela 1. O azeite de dendê é extraído do fruto da palmeira e não é submetido a qualquer processo de refino (BRASIL, 1999), o que explica as altas porcentagens de ácidos graxos livres $(2,50 \%)$ e compostos polares totais $(8,60 \%)$ presentes na amostra inicial.

A influência dos fatores relação $\mathrm{S} / \mathrm{V}$ e tempo de fritura nos resultados das determinações analíticas é apresentada na Tabela 2. O fator relação $\mathrm{S} / \mathrm{V}$ foi significativo $(\mathrm{P}<0,01)$ apenas nos índices de peróxidos e de refração, enquanto que o tempo de fritura apresentou influência significativa $(\mathrm{P}<0,01)$ em todas as determinações analíticas.

A interação entre relação $S / V$ e tempo de fritura foi significativa $(\mathrm{P}<0,01)$ somente para o índice de peróxidos, o que significa que para este parâmetro os efeitos das diferentes relações $\mathrm{S} / \mathrm{V}$ dependem dos tempos de fritura utilizados e os efeitos dos tempos de fritura dependem da relação S/V utilizada. Desta forma, procedeu-se ao desdobramento dessa interação cujos resultados encontram-se na Tabela 3. Comparando-se as duas relações $\mathrm{S} / \mathrm{V}$, verificou-se diferenças significativas $(\mathrm{P}<0,05)$ entre todas as frituras sendo que as amostras com relação $\mathrm{S} / \mathrm{V}$ $1,0 \mathrm{~cm}^{-1}$ apresentaram valores superiores àquelas com relação $S / V \quad 0,5 \mathrm{~cm}^{-1}$. Constatou-se que quanto maior a relação $\mathrm{S} / \mathrm{V}$, isto é, maior a exposição ao ar, maior a formação de hidroperóxidos, visto que os peróxidos são originados pelas reações oxidativas devido à presença do oxigênio do ar, catalisadas pelo aumento de temperatura.

Durante o processo de oxidação o índice de peróxidos atinge um valor máximo e, em seguida, declina. Este pico é alcançado após cerca de 20 horas de utilização do óleo em frituras (CUESTA et al., 1991; LIMA, 1994). De acordo com a Tabela 3, o índice de peróxidos aumentou ao longo do processo de fritura nas duas relações $S / V$, sendo verificadas diferenças significativas $(\mathrm{P}<0,05)$ entre os diferentes tempos de fritura. Somente os tempos de fritura 1,25 e 2,25 horas $\left(\mathrm{S} / \mathrm{V} 0,5 \mathrm{~cm}^{-1}\right)$ e 3,$25 ; 4,25$ e 6,25 horas $\left(\mathrm{S} / \mathrm{V} 1,0 \mathrm{~cm}^{-1}\right)$ não diferiram significativamente.

TABELA 1-Características físico-químicas originais da mistura azeite de dendê-óleo de soja (1:1).

\begin{tabular}{lc}
\hline Características físico-químicas & Valores médios \\
\hline Índice de peróxidos $(\mathrm{meq} / \mathrm{kg})$ & 5,00 \\
Compostos polares totais $(\%)$ & 8,60 \\
Ácidos graxos livres $(\%)$ & 2,50 \\
Índice de refração $\left(40^{\circ} \mathrm{C}\right)$ & 1,4621 \\
\hline
\end{tabular}

TABELA 2 -Análises de variância para as determinações de índice de peróxidos (IP), compostos polares totais (CPT), ácidos graxos livres (AGL) e índice de refração (IR) na mistura azeite de dendê-óleo de soja (1:1).

\begin{tabular}{|c|c|c|c|c|c|}
\hline \multirow[b]{2}{*}{ Causas de Variação } & \multirow[b]{2}{*}{ G.L. } & \multicolumn{4}{|c|}{ Quadrados Médios } \\
\hline & & IP & CPT & AGL & $\mathbf{I R}^{1}$ \\
\hline $\mathrm{S} / \mathrm{V}$ & 1 & $659,8436 * *$ & $2,0000^{\mathrm{ns}}$ & $0,0060^{\mathrm{ns}}$ & $0,0903 * *$ \\
\hline Tempo de fritura & 7 & $192,1742 * *$ & $199,5625 * *$ & $0,1367 * *$ & $1,2189 * *$ \\
\hline S/V x Tempo de fritura & 7 & $22,6871^{* *}$ & $1,7364^{\mathrm{ns}}$ & $0,0486^{\mathrm{ns}}$ & $0,0110^{\mathrm{ns}}$ \\
\hline Resíduo & 16 & 0,0531 & 1,7686 & 0,0034 & 0,0047 \\
\hline Desvio Padrão & & 0,2305 & 1,3299 & 0,0579 & 0,0685 \\
\hline Coeficiente de Variação (\%) & & 1,22 & 6,87 & 2,04 & 0,005 \\
\hline
\end{tabular}

Ciênc. agrotec., Lavras, v. 30, n. 4, p. 724-730, jul./ago., 2006 
TABELA 3 - Desdobramento da interação relação $\mathrm{S} / \mathrm{V}$ x tempo de fritura para os dados de índice de peróxidos obtidos na mistura azeite de dendê-óleo de soja (1:1) durante fritura de batatas.

\begin{tabular}{|c|c|c|c|c|c|c|c|c|}
\hline \multirow{2}{*}{$\begin{array}{c}\mathrm{S} / \mathrm{V} \\
\left(\mathrm{cm}^{-1}\right)\end{array}$} & \multicolumn{8}{|c|}{ Tempo de fritura (horas) } \\
\hline & 0,25 & 1,25 & 2,25 & 3,25 & 4,25 & 5,25 & 6,25 & 7,25 \\
\hline 0,5 & $8,05^{\mathrm{gB}}$ & $9,10^{\mathrm{fB}}$ & $9,29^{\mathrm{fB}}$ & $10,55^{\mathrm{eB}}$ & $15,10^{\mathrm{dB}}$ & $17,27^{\mathrm{cB}}$ & $20,98^{\mathrm{bB}}$ & $24,25^{\mathrm{aB}}$ \\
\hline 1,0 & $8,79^{\mathrm{fA}}$ & $15,67^{\mathrm{eA}}$ & $20,06^{\mathrm{dA}}$ & $25,23^{\mathrm{cA}}$ & $25,88^{\mathrm{cA}}$ & $31,00^{\mathrm{bA}}$ & $25,48^{\mathrm{cA}}$ & $35,13^{\mathrm{aA}}$ \\
\hline
\end{tabular}

a, b... (linha) em cada $\mathrm{S} / \mathrm{V}$, médias de tempos de fritura seguidas de mesma letra minúscula não diferem entre si pelo teste de Tukey $(\mathrm{P}>0,05)$;

A, B (coluna) em cada tempo de fritura, médias de $S / V$ seguidas de mesma letra maiúscula não diferem entre si pelo teste de Tukey $(\mathrm{P}>0,05)$.

Alguns estudos utilizando óleos de soja, algodão, girassol, milho e palma em frituras de alimentos demonstraram que o índice de peróxidos apresentou um comportamento instável ao longo dos tempos de fritura, oscilando entre um aumento e uma diminuição (CORSINI, 2004; DEL-RÉ, 2003; MALACRIDA \& JORGE, 2005). Isto se deve ao fato de que nas temperaturas utilizadas em processos de fritura, os hidroperóxidos se decompõem rapidamente dando origem a produtos secundários de oxidação (CUESTA et al., 1991). Assim, o índice de peróxidos não é considerado o melhor parâmetro analítico para ser utilizado na avaliação de óleos e gorduras de fritura, sendo aplicável somente em estágios iniciais de oxidação, uma vez que não quantifica produtos de oxidação secundária (SANIBAL \& MANCINI-FILHO, 2002).

Segundo Monferrer \& Villalta (1993), óleos de fritura com valores de índice de peróxidos superiores a $15 \mathrm{meq} / \mathrm{kg}$ são considerados altamente alterados e devem ser descartados. Neste trabalho, valores acima deste limite foram alcançados após 4,25 e 1,25 horas de fritura nas relações $\mathrm{S} / \mathrm{V}$ 0,5 e $1,0 \mathrm{~cm}^{-1}$, respectivamente. Adotando-se esta recomendação observa-se que a mistura azeite de dendê-óleo de soja (1:1) pode ser utilizada por mais tempo em frituras descontínuas quando a relação $\mathrm{S} / \mathrm{V}$ é menor.

Os compostos polares totais constituem os produtos de degradação dos triglicerídios: ácidos graxos livres, ácidos graxos oxidados, polímeros, etc, originados de alterações hidrolíticas, oxidativas e térmicas do óleo de fritura (MÁRQUEZ-RUIZ et al., 1990).

A relação $S / V$ não apresentou influência sobre as porcentagens de compostos polares totais, sendo que não foram verificadas diferenças significativas $(P>0,05)$ entre as médias das frituras com relação $\mathrm{S} / \mathrm{V} 0,5 \mathrm{~cm}^{-1}(19,62 \%) \mathrm{e}$ $1,0 \mathrm{~cm}^{-1}(19,12 \%)$. Independentemente da relação $\mathrm{S} / \mathrm{V}$, os valores de compostos polares totais aumentaram ao longo do processo de fritura, principalmente após 5,25 horas de fritura (Tabela 4).

Del-Ré et al. (2003) estudaram o comportamento do óleo de soja em frituras descontínuas de batatas palito à temperatura de $180^{\circ} \mathrm{C}$ e relações $\mathrm{S} / \mathrm{V}$ de 0,$2 ; 0,4$ e $0,6 \mathrm{~cm}^{-1}$. Os autores verificaram aumento na porcentagem de compostos polares totais com o tempo de fritura e a relação $\mathrm{S} / \mathrm{V}$.

Muitos países adotaram a quantificação dos produtos totais de alteração como método padrão para a avaliação de óleos e gorduras em processos de fritura e estabeleceram em $25 \%$ a quantidade máxima de compostos polares totais que podem estar presentes nestes óleos (FIRESTONE et al., 1991). Neste trabalho, foram verificados valores acima de $25 \%$ de compostos polares após 6,25 horas de utilização do óleo.

Conforme a Tabela 4 , as médias de ácidos graxos livres nas relações $\mathrm{S} / \mathrm{V} 0,5$ e $1,0 \mathrm{~cm}^{-1}$ não diferiram significativamente, o que demonstra que o aumento na relação $S / V$ não influenciou na formação de ácidos graxos livres. Independentemente da relação $\mathrm{S} / \mathrm{V}$, houve um aumento na porcentagem de ácidos graxos livres com o tempo de fritura, sendo que o tempo de fritura 7,25 horas diferiu significativamente $(P<0,05)$ das demais.

A velocidade de formação de ácidos graxos livres é influenciada por vários fatores entre os quais a temperatura de fritura, a quantidade de água liberada pelo alimento que está sendo frito, os números de aquecimento e resfriamento do óleo e a quantidade de partículas queimadas proveniente do alimento e acumuladas no recipiente (LAWSON, 1995). Além disso, a acidez livre encontrada no óleo não reflete apenas os ácidos graxos formados durante o processo de fritura como também, aqueles inicialmente presentes no óleo antes do aquecimento e extraídos dos alimentos que estão sendo fritos (MASSON et al., 1997). 
TABELA 4 - Valores médios de compostos polares totais (CPT), ácidos graxos livres (AGL) e índice de refração (IR) da mistura azeite de dendê-óleo de soja (1:1) durante frituras de batatas.

\begin{tabular}{|c|c|c|c|}
\hline \multirow[b]{2}{*}{ Fatores } & \multicolumn{3}{|c|}{ Valores médios* } \\
\hline & CPT (\%) & AGL (\%) & IR $\left(40^{\circ} \mathrm{C}\right)$ \\
\hline \multicolumn{4}{|l|}{$\mathrm{S} / \mathrm{V}\left(\mathrm{cm}^{-1}\right)$} \\
\hline 0,5 & $19,62^{\mathrm{a}}$ & $2,85^{\mathrm{a}}$ & $1,4629^{\mathrm{a}}$ \\
\hline 1,0 & $19,12^{\mathrm{a}}$ & $2,85^{\mathrm{a}}$ & $1,4628^{\mathrm{b}}$ \\
\hline \multicolumn{4}{|l|}{ Tempo de fritura (horas) } \\
\hline 0,25 & $10,43^{\mathrm{f}}$ & $2,53^{\mathrm{e}}$ & $1,4622^{\mathrm{g}}$ \\
\hline 1,25 & $12,50^{\mathrm{ef}}$ & $2,65^{\mathrm{de}}$ & $1,4622^{\mathrm{g}}$ \\
\hline 2,25 & $14,37^{\mathrm{de}}$ & $2,77^{\mathrm{cd}}$ & $1,4625^{\mathrm{f}}$ \\
\hline 3,25 & $17,29^{\mathrm{cd}}$ & $2,85^{\mathrm{bc}}$ & $1,4627^{\mathrm{e}}$ \\
\hline 4,25 & $20,27^{\mathrm{bc}}$ & $2,91^{\mathrm{bc}}$ & $1,4629^{d}$ \\
\hline 5,25 & $22,56^{\mathrm{b}}$ & $2,93^{\mathrm{b}}$ & $1,4631^{\mathrm{c}}$ \\
\hline 6,25 & $27,15^{\mathrm{a}}$ & $2,96^{\mathrm{b}}$ & $1,4634^{\mathrm{b}}$ \\
\hline 7,25 & $30,38^{\mathrm{a}}$ & $3,12^{\mathrm{a}}$ & $1,4637^{\mathrm{a}}$ \\
\hline
\end{tabular}

* Em cada fator, médias seguidas de mesma letra não diferem entre si pelo teste de Tukey $(\mathrm{P}>0,05)$.

Tyagi \& Vasishtha (1996) também verificaram, em diversos óleos vegetais, aumento na porcentagem de ácidos graxos livres com o tempo de fritura. Os autores atribuíram este aumento à ocorrência de reações hidrolíticas e também à presença de grupos carboxílicos nos polímeros formados durante o processo de fritura.

Todas as amostras apresentaram porcentagens de ácidos graxos livres acima de $1 \%$, valor este considerado limite para a utilização de óleos de fritura (FIRESTONE et al., 1991). Os elevados valores de acidez livre encontrados neste trabalho devem-se à condição inicial do óleo, uma vez que a amostra original já apresentava um valor de acidez livre elevado (2,50\%), não sendo indicativos de alto grau de degradação durante a fritura.

Com relação ao índice de refração verificou-se diferença significativa $(\mathrm{P}<0,05)$ entre as médias das duas relações $S / V$, sendo que a média da relação $S / V 0,5 \mathrm{~cm}^{-1}(1,4629)$ foi superior a da relação $S / V 1,0 \mathrm{~cm}^{-1}(1,4628)$. Nos diferentes tempos de fritura observou-se um aumento no índice de refração após 1,25 horas de utilização do óleo (Tabela 4).

As variações do índice de refração são paralelas à formação de polímeros nos óleos e gorduras aquecidos (GONZÁLEZ-QUIJANO \& DOBARGANES, 1988). Os resultados obtidos indicam um aumento da oxidação e conseqüente polimerização da mistura azeite de dendêóleo de soja ao longo do processo de fritura.

Outros autores verificaram resultados semelhantes aos encontrados neste trabalho para o índice de refração. Damy \& Jorge (2003), em estudos sobre o comportamento do óleo de soja refinado e da gordura vegetal hidrogenada em frituras descontínuas de batatas, observaram aumento do índice de refração com as sucessivas frituras e o grau de insaturação dos óleos.

\section{CONCLUSÕES}

- Todas as determinações analíticas realizadas na mistura azeite de dendê-óleo de soja (1:1) foram influenciadas pelo tempo de fritura, verificando-se aumentos significativos destes parâmetros ao longo do processo de fritura;

- Os índices de peróxidos e de refração da mistura azeite de dendê-óleo de soja (1:1) foram influenciados pela relação superfície/volume;

- Diante das condições do processo, recomenda-se o uso da mistura azeite de dendê-óleo de soja (1:1) em frituras até 5,25 horas de aquecimento, pois a partir deste tempo de fritura observou-se alterações acima dos limites recomendados para compostos polares totais (CPT $\geq 25 \%$ ).

\section{REFERÊNCIAS BIBLIOGRÁFICAS}

AMERICAN OILCHEMISTS' SOCIETY. Official methods and recommended practices of the American Oil Chemists' Society. Champaign, 1993.

BRASIL. Resolução n. 482, de 23 de setembro de 1999. Aprova o Regulamento Técnico para fixação de identidade e qualidade de óleos e gorduras vegetais. Diário Oficial da União, Brasília, v. 196, p. 82-87, 13 out. 1999. Seção I. 
CORSINI, M. S. Medidas dos teores de tocoferóis e estabilidade oxidativa em óleos de fritura. 2004. $153 \mathrm{f}$. Dissertação (Mestrado em Engenharia e Ciência de Alimentos) - Universidade Estadual Paulista, São José do Rio Preto, 2004.

CUESTA, C.; SÁNCHEZ-MUNIZ, F. J.; HERNANDÉZ, I.; VARELA, L. S. Modificaciones de un aceite de oliva durante las frituras sucesivas de patatas: correlaciones entre distintos índices analíticos y de evaluación global de la degradación. Revista Agroquímica Tecnologia de Alimentos, [S.1.], v. 31, n. 4, p. 523-531, 1991.

DAMY, P. C.; JORGE, N. Determinações físico-químicas do óleo de soja e da gordura vegetal hidrogenada durante o processo de fritura descontínua. Brazilian Journal Food Technology, São Paulo, v. 6, n. 2, p. 251-257, 2003.

DEL-RÉ, P. V. Comportamento de óleos vegetais em frituras descontínuas de produtos pré-fritos congelados. 2003. 121 f. Dissertação (Mestrado em Engenharia e Ciência de Alimentos) - Universidade Estadual Paulista, São José do Rio Preto, 2003.

DEL-RÉ, P. V.; COLTRO, A. L.; MANENTE, J. C. P. P.; MARTI, G. E.; JORGE, N. Influência da relação superfície/volume em frituras de batata palito. Revista do Instituto Adolfo Lutz, São Paulo, v. 62, n. 3, p. 213219, 2003.

DOBARGANES, M. C.; PÉREZ-CAMINO, M. C.; GONZÁLEZ-QUIJANO, R. G. Métodos analíticos de aplicación en grasas calentadas: I. determinación de ésteres metílicos no alterados. Grasas y Aceites, Sevilha, v. 35, n. 3, p. 172-177, 1984.

FIRESTONE, D.; STIER, R. F.; BLUMENTHAL, M. M. Regulation of frying fats and oils. Food Technology, Oxford, v. 45, n. 2, p. 90-94, 1991.

FIRESTONE, D. Regulation of frying fat and oil. In: PERKINS, E. G.; ERICKSON, M. D. (Eds.). Deep frying: chemistry, nutrition and practical applications. Champaign: AOCS, 1996. p. 323-334.

GOMES, F. P. Curso de estatística experimental. 14. ed. Piracicaba: Nobel, 2000. 477 p.
GONZÁLEZ-QUIJANO, R. G.; DOBARGANES, M. C. Analytical procedures for the evaluation of used frying fats. In: VARELA, G.; BENDER, A. E.; MORTON, I. A. (Eds.). Frying foods: principles, changes, new approaches. Chichester: E. Horwood, 1988. p. 141-154.

LAWSON, H. Food oils and fats: technology, utilization and nutrition. New York: Chapman \& Hall, 1995. 339 p.

LIMA, J. R. Avaliação da qualidade de óleo de soja utilizado para fritura. 1994. 54 f. Dissertação (Mestrado em Tecnologia de Alimentos) - Universidade Estadual de Campinas, Campinas, 1994.

MALACRIDA, C. R.; JORGE, N. Alterações do óleo de soja em frituras: efeitos da relação superfície/volume e do tempo de fritura. Higiene Alimentar, São Paulo, v. 19, n. 129, p. 25-31, 2005.

MÁRQUEZ-RUIZ, G.; PÉREZ-CAMINO, M. C.; DOBARGANES, M. C. Evaluación nutricional de grasas termoxidadas y de fritura. Grasas y Aceites, Sevilha, v. 41, n. 6, p. 432-439, 1990.

MASSON, L.; ROBERT, P.; ROMERO, N.; IZAURIETA, M.; VALENZUELA, S.; ORTIZ, J.; DOBARGANES, M. C. Comportamiento de aceites poliinsaturados en la preparación de patatas fritas para consumo inmediato: formación de nuevos compuestos y comparación de métodos analíticos. Grasas y Aceites, Sevilha, v. 48, n. 5 , p. 273-281, 1997.

MONFERRER, A.; VILLALTA, J. La fritura desde un punto de vista práctico. Alimentos Equipos Tecnologia, [S.1.], v. 21, n. 3, p. 85-90, 1993.

NAWAR, W. W. Lipids. In: FENNEMA, O. R. (Ed.). Food chemistry. 3. ed. New York: M. Dekker, 1996. p. 225-319.

ROSSELL, J. B. Classical analysis of oils and fats. In: HAMILTON, R. J.; ROSSELL, J. B. (Eds.). Analysis of oils and fats. New York: Elsevier Applied Science, 1986. p. 1-90.

SANIBAL, E. A.; MANCINI-FILHO, J. Alterações físicas, químicas e nutricionais de óleos submetidos ao processo de fritura. Food Ingredient South American, [S.1.], v. 18, p. 64-71, 2002. 
TYAGI, V. K.; VASISHTHA, A. K. Changes in the characteristics and composition of oils during deep-fat frying. Journal of the American Oil Chemists' Society, Chicago, v. 73, n. 4, p. 499-506, 1996.
WALTKING, A. E.; WESSELS, H. Chromatographic separation of polar and non-polar components of frying fats. Journal of the Association Office Analytical Chemists, Arlington, v. 64, n. 6, p. 1329-1330, 1981.

Ciênc. agrotec., Lavras, v. 30, n. 4, p. 724-730, jul./ago., 2006 